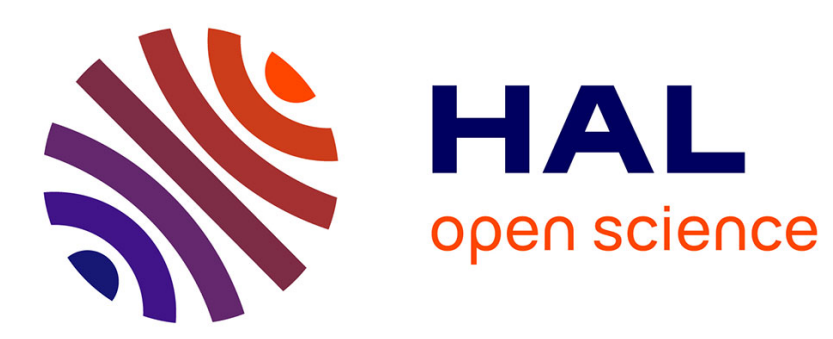

\title{
Enhanced EDX images by fusion of multimodal SEM images using pansharpening techniques
}

\author{
Gianni Franchi, Jesus Angulo, Maxime Moreaud, Loïc Sorbier
}

\section{To cite this version:}

Gianni Franchi, Jesus Angulo, Maxime Moreaud, Loïc Sorbier. Enhanced EDX images by fusion of multimodal SEM images using pansharpening techniques. Journal of Microscopy, 2018, 269 (1), pp.94 - 112. 10.1111/jmi.12612 . hal-01688728

\section{HAL Id: hal-01688728 https://hal.science/hal-01688728}

Submitted on 19 Jan 2018

HAL is a multi-disciplinary open access archive for the deposit and dissemination of scientific research documents, whether they are published or not. The documents may come from teaching and research institutions in France or abroad, or from public or private research centers.
L'archive ouverte pluridisciplinaire HAL, est destinée au dépôt et à la diffusion de documents scientifiques de niveau recherche, publiés ou non, émanant des établissements d'enseignement et de recherche français ou étrangers, des laboratoires publics ou privés. 


\title{
Enhanced EDX images by fusion of multimodal SEM images using pansharpening techniques
}

\author{
Gianni Franchi ${ }^{1}$, Jesus Angulo ${ }^{1}$, Maxime Moreaud ${ }^{2}$, Loïc Sorbier ${ }^{2}$ \\ ${ }^{1}$ MINES Paristech, PSL Research University, CMM-Centre de Morphologie Mathématique, France, \\ ${ }^{2}$ IFP Energies Nouvelles, BP 3, 69360, Solaize, France
}

\begin{abstract}
The goal of this paper is to explore the potential interest of image fusion in the context of multimodal scanning electron microscope (SEM) imaging. In particular, we aim at merging the backscattered electron images that usually have a high spatial resolution but do not provide enough discriminative information to physically classify the nature of the sample, with energy-dispersive X-ray spectroscopy (EDX) images that have discriminative information but a lower spatial resolution. The produced images are named enhanced EDX. To achieve this goal, we have compared the results obtained with classical pansharpening techniques for image fusion with an original approach tailored for multimodal SEM fusion of information. Quantitative assessment is obtained by means of two SEM images and a simulated dataset produced by a software based on PENELOPE.
\end{abstract}

Keywords : fusion of images; SEM images; multivariate image processing, multimodalities

\section{Introduction}

Scanning electron microscope (SEM) is a versatile imaging tool that allows to acquire images with various detectors. Images formed by secondary electrons (SE) reveals mainly topographic contrasts, images formed by backscattered electrons (BSE) indicates local mean atomic number, whereas X-ray maps contains local elemental composition. Coupling a SEM with an energy dispersive spectrometer (EDS) leads to so called energy-dispersive X-ray spectrometry in the SEM (SEM-EDX). If the full X-ray spectrum is recorded on each scanned pixel, SEM-EDX produces spectral images. Typical SEM-EDX spectra are few millions pixels times few thousands of energy channels. The set of the images produced by the different SEM detectors can be seen as a multimodal image. Then, the various images can be processed independently or in a combined way. It seems also natural to consider that an improved processing would be obtained by combining the information present in the different modalities; obviously, that is true in the case where the modalities are "compatible". This process of image combination can be seen as a practical case of the theory of information fusion. 
More generally, the fusion of information can be seen either as the search for optimal representation including all relevant information sources [Piella2003] or as the search for algorithms making use of information from different modalities [Wald1999].

In the context of multimodal SEM, we decided to focus on a specific problem. We aim at merging the backscattered electron images that usually have a high spatial resolution, a good signal to noise ratio but do not provide enough discriminative information to physically classify the nature of the sample, with energy-dispersive X-ray spectroscopy images that have discriminative information but a lower spatial resolution and signal to noise ratio. Such image fusion is rather restricted to flat and polished sample where both EDX and backscattered electron images are not affected by topographical effects. Indeed, for non-flat samples, topographic effects can be very strong on EDX images and abundance retrieval is dependent on the local geometry.

This problem is similar in some ways to the so-called pansharpening [Laben2000, King2001], which is well known in colour, multispectral and hyperspectral imaging. However there are also some significant differences. The first one is that in the case of classical image pansharpening, the panchromatic image (i.e., the image at the nominal spatial resolution) has a good correlation with the colour or multi/hyperspectral image. Even better, in some cases the "panchromatic image" is contained partly at some wavelengths (or linear combination of them) from the multi/hyperspectral one. In our case, the information between the two SEM modalities that are considered is not basically correlated. Moreover, we work with abundance maps extracted from the energy-dispersive spectroscopy images, since the raw EDS spectral image are of very high dimension and of very sparse nature.

As usual in multimodal SEM, we work with images perfectly registered. Although these images are spatially registered, it can be noticed that there are a number of potential artefacts which can appear when merging their underlying information sources. The origin of these artefacts is the fact that information can be structurally different, in the sense of the values of intensities, local contrast, presence of contours, etc. can be different between backscattered electron images and energy-dispersive spectroscopy. All these phenomena may degrade the quality of the fusion. A detailed list of the potential artefacts classicaly considered in image pansharpening can be found in [Thomas2008]. It seems essential to deal with these problems in order to produce image fusion without major artifacts.

Up to the best of our knowledge, the literature on SEM image fusion is quite limited. On [Wortmann2009, Angelov20016] Atomic Force Microscopy and SEM are combined to provide a view of the topography and material properties of the sample. In [Milillo2008] . Thus to initiate our work, we decided to take inspiration from the work of fusion of information on multi/hyperspectral imaging which is much more abundant and especially on pansharpening techniques [Vivone2015, Loncan2015]. The rest of the paper is organized as follows. After providing a description of the SEM dataset used and a summary of the most frequently used pansharpening algorithms, we introduce a new approach grounded on the bilateral filtering framework, which has been conceived in the particular case of SEM images fusion. A extensive 
quantitative assessment of the different algorithms is achieved to motivate discussion and conclusions.

\section{Materials and methods}

Multimodal SEM imaging. SEM is able to produce high resolution images from the surface of a sample by means of an extremely small electron beam, which is focused at a point of the sample using the electromagnetic field of an objective lens [Reimer1998]. When electrons of the beam hit the specimen surface, electron-matter interactions may produce secondary particles that are detected by adequate sensors. These secondary particles can produce several types of images, as shown in Figure 1. In this work we focus primarily on two types of images :

- Images formed by backscattered electrons (BSE);

- Images formed by electron-induced X-ray detected by EDS

Hence, we do not consider the Secondary electrons image, which is quite classic in SEM, due to the fact that it is less correlated with the two others modalities BSE and EDX. The different modalities acquired by the SEM provide different physical information about the sample, and consequently it can be interesting to merge them. This principle corresponds to the idea of SEM image information fusion.

SEM datasets. SEM fusion methods discussed in this paper has been assessed using two real datasets and a simulate one.

The first dataset is composed of an EDX image of size $1024 \times 768$ pixels and 2024 levels of energy. Thus the image is a data cube $1024 \times 768 \times 2024$ pixels. As an EDX image, it is naturally corrupted by a Poisson process and of extremely sparse nature, i.e., many energy levels are zero. This image is from a sample composed of iron, copper, aluminium and oxygen. This image has been acquired with a Zeiss Supra 40 SEM fitted with a $10 \mathrm{~mm}^{2}$ Brucker X-Flash 4010 EDS spectrometer. Beam energy was $15 \mathrm{keV}$, probe current $0.75 \mathrm{nA}$ and dwell time $912 \mu$ s resulting in a $717 \mathrm{~s}$ long acquisition time. To estimate the abundance map of each of these physical elements, a Gaussian model near each peak is fitted as EDS peak shapes are very close to a Gaussian shape due to electron-hole formation statistics in the detector [Scholze2009, Bauer1978]. Since there are 6 detectable peaks at 6 different energies, 6 abundance maps are obtained. In the following of the paper, the multivariate image of physical abundances is called the "multispectral image". In addition to that, the BSE image of the same sample at the same spatial resolution is also available. To downscale the abundance maps, the experimental protocol that we followed is similar to the one proposed by the authors of [Loncan2015]. We have degraded the multispectral image by applying a Gaussian blur, then we have downsampled this image by a factor $s=5$. The second dataset 
is composed of an EDX image of size $1024 \times 704$ pixels and 2024 levels of energy and the corresponding BSE image. This image has been acquired with a FEI Nova nanoSEM fitted with a $80 \mathrm{~mm}^{2}$ Oxford Instruments X-Max EDS spectrometer. Beam energy was $10 \mathrm{keV}$, probe current $0.8 \mathrm{nA}$ and dwell time $3.2 \mathrm{~ms}$ per pixel resulting in a $2307 \mathrm{~s}$ long acquisition time. This image is from a sample composed of aluminum, oxygen, vanadium and nickel. In this case, 4 abundance maps were obtained using the same model and algorithm. Downscaled images of the abundance maps image were produced too.

In order to assess the accuracy of the algorithms, EDX images have been simulated by the Monte-Carlo method by a dedicated software based on the PENELOPE package [Sempau1997, Sempau2003, Salvat2006]. First, the response of the EDS detector of the Nova NanoSEM has been characterized by measuring the full width at half maximum (FWHM) for different peak energies. The dependence on peak energy $E$ of the standard deviation of the Gaussian peaks $\sigma(E)$ was obtained by least-square fitting and gave :

$$
\sigma(E)=\sqrt{0.4403 E+337.04}
$$

with $E$ in $\mathrm{eV}$. The efficiency $\epsilon(E)$ of the detector was modeled from its geometry following the same approach proposed by Limandri et al. [Limandri2013]. Analog electrons trajectories $(C 1=C 2=0 ; W C C=W C R=0)$ were generated with the PENELOPE package. As Xray emission is an unusual process, both characteristic and Bremsstrahlung photon emission were enhanced by interaction forcing by a factor $F$ with the help of the build-in functions of PENELOPE. Sample and detector geometries were handled by the PENGEOM package. The detector is annular at a $35^{\circ}$ take-off angle to the surface. Any photon hitting the detector was considered detected with a probability $\epsilon(E)$. Each detected photon of energy $E$ was registered in an energy channel distributed following a Gaussian law of average $E$ and standard deviation $\sigma(E)$. Backscattered electrons were also registered to obtain a simulated BSE image. The image simulations were performed with 10000 electrons trajectories for each pixel with varying image size and forcing factor $F$. Varying $F$ allowed to tune the intensity of Poisson noise of the simulated images. Two images of $1024 \times 1024$ pixels with $F=1$ and $F=100$ and three images of $256 \times 256$ pixels with $F=1, F=10$ and $F=100$ were simulated. Typical simulation time was about 10 days for a $1024 \times 1024$ pixels image on an 8 processors working station. Figure 2 depicts the ground truth sample used in the simulation: it is composed of regular geometric shapes of pure and binary composition with low mean atomic number element $\left(\mathrm{Al}_{2} \mathrm{O}_{3}, \mathrm{SiO}_{2}\right)$, medium $(\mathrm{Fe}, \mathrm{Co}, \mathrm{Ni})$ and high $(\mathrm{Pt})$. Figure 3 provides the simulated total backscattered electrons image. It is worth noticing that phases with close mean atomic number $\left(\mathrm{Al}_{2} \mathrm{O}_{3}\right.$ and $\mathrm{SiO}_{2} ; \mathrm{Fe}$, Co and $\left.\mathrm{Ni}\right)$ are hard to be distinguished in the BSE image. In Figure 4 the different simulated EDX map are represented. 


\section{State-of-the-art}

Before presenting the different image fusion methods, let us introduce the notation used in the following, similar to [Loncan2015].

First, from a mathematical viewpoint, a multispectral image (abundance maps) is considered as a function $H S$ defined by

$$
H S:\left\{\begin{array}{l}
E \rightarrow \mathbb{R}^{D} \\
x \mapsto v_{i}
\end{array}\right.
$$

where $D$ is the number of abundance maps and $E$ is the image domain (support space of pixels). This multivariate image can be also seen as set of $D$ grey-scale images. We note $H S$ a multispectral image at a low resolution. Let $\widetilde{H S} \in \mathbb{R}^{N_{1} \times N_{2} \times D}$ be a interpolated multispectral image whose spatial dimensions are $N_{1}, N_{2}$, which in our case corresponds to BSE image dimensions. Let $R \in \mathbb{R}^{N_{1} \times N_{2}}$ be just the BSE image. We denote by $\widehat{H S} \in \mathbb{R}^{N_{1} \times N_{2} \times D}$ the multispectral image of the EDS abundances enhanced with the BSE image information, where $H S_{k}$ is the $k$-th abundance map of image $H S$.

There are essentially three families of pansharpening techniques which are detailed as follows.

\subsection{Component substitution methods (CS)}

The purpose of information fusion techniques is to find the function $\phi$ satisfying

$$
\widehat{H S}=\phi(\widetilde{H S}, R)
$$

where $\widehat{H S}$ is "optimal" in a certain sense. The particular notion of optimality is precised below. Component substitution methods (CS) are based on the projection of the image $H S$ into another space, separating the spatial information from the spectral one. The spatial information, i.e., contrast and contours between different objects, is often concentrated in a single grey-scale image. The main step of CS consists in replacing the image containing the spatial information by the spatially high resoluted image $R$. It is based on the assumption that the image containing only spatial information is highly correlated with $R$. Once the corrections are done, the data are projected back to the initial space. This approach is global and therefore corrects all pixels with a single rule. The strength of this technique is its speed, however it depends on the fact that the image containing the spatial information of $H S$ which must be similar in range and intensity distribution to $R$. If not, the merged image may have strong distortions. In our particular case, the BSE image has information which is not comparable with EDS images, that explains the artefacts which appears in our results, see Section 5.

The problem can be formulated mathematically as follows [Loncan2015]:

$$
\widehat{H S}_{k}=\widetilde{H S}_{k}+g_{k}\left(R-I_{L}\right)
$$


where $g_{k}$ corresponds to a corrective coefficient for the band $k$ and $I_{L}$ is the image containing all high resolution spatial information, i.e., typically

$$
I_{L}=\sum_{i=1}^{D} w_{i} \widetilde{H S}_{i}
$$

where $w_{i}$ is a coefficient depending on the degree of the spatial information of the spectral band $i$. Now let us present in a more detail way the most popular CS techniques.

\subsubsection{CS by PCA.}

Principal Component Analysis (PCA) [Pearson1901, Hotelling1993, Chavez1991] is a wellknown dimensionality reduction technique, which aims at finding an orthonormal basis that maximizes the variance of the data. Thus, the data projected into this space summarize the statistically significant information. The fundamental assumption of this pansharpening technique is that the first component focuses all relevant spatial information while the other components contain secondary spatial and spectral contrast features. Using the CS model, the first principal component of $\widetilde{H S}$ is $I_{L}$, and $w_{i}$ is the first column of the inverse transform. Note that this is not exactly the $R$ which is used in the Eq. (2), since the version of $R$ should be histogram equalized with respect to $I_{L}$.

\subsubsection{CS by Gram-Schmidt decomposition (GS).}

The Gram-Schmidt (GS) technique is based on an orthogonal decomposition, which was invented by Kodak [Laben2000]. First, instead of considering only the cube $\widetilde{H S}$, one starts with the tensor composed of the concatenation of $\widetilde{H S}$ and $R$, thus of $D+1$ bands. Then, an orthogonal decomposition is performed on this new tensor. We find the vector of the basis that corresponds to the spatial information and it is replaced by $R$. Finally a reverse procedure is done to return to the initial representation space. The components of the Gram-Schmidt basis being orthogonal, the spatial information is expected to be orthogonal to all the other information sources. That is why the spatial information is, in theory, gathered in a single vector. In practice this is not always the case, especially if $R$ contains dense information. Moreover this technique can be problematic in the case of multimodal SEM images since a significant orthogonal basis from EDX + BSE cannot be easily obtained without adding a kind of sparsity constraint.

\subsection{Multiresolution analysis methods (MRA)}

MRA techniques are founded on the application of a low pass filter to $R$, sometimes under different resolutions. Then, details are injected on $\widetilde{H S}$ thanks to the residue of $R_{L}$ and $R$, which represents the high frequences of $R$. A mathematical formulation of this type of 
technique is written as [Ranchin2000, Tu2001]:

$$
\widehat{H S}_{k}=\widetilde{H S}_{k}+G_{k} \otimes\left(R-R_{L}\right)
$$

where $G_{k}$ corresponds to a corrective coefficient matrix for the band $k$ and $\otimes$ is the multiplication term by term. Moreover, as mentioned above, $R-R_{L}$ represents the details that are injected in the low resolution image. Such family of techniques depends mainly on the type of decomposition performed for $R_{L}$ and the diagonal matrix of gains $G_{k}$. Different kind of filters can be used for $R_{L}$ : Gaussian filters, wavelets, mathematical morphology operators, etc. There are therefore many possibilities. Two techniques are now detailed.

\subsubsection{MRA by Smoothing Filter-based Intensity Modulation (SFIM).}

The technique [Liu2000] involves the use of a single low pass filter, noted $H_{l p}$, applied to $R$ to get $R_{L}$. The enhancement is then obtained as:

$$
\widehat{H S}_{k}=\widetilde{H S}_{k}+G_{k} \otimes\left(R-R * H_{l p}\right),
$$

with

$$
G_{k}=\widetilde{H S}_{k} \odot R * H_{l p}
$$

where $\odot$ is the division term by term. To achieve the low pass filter, a simple average filter is typically used. The formula can be simplified thanks to the coefficient matrix to obtain:

$$
\widehat{H S}_{k}=R \otimes\left(\widetilde{H S}_{k} \odot R_{l}\right) .
$$

Thus it involves that the information of $R$ was modulated by the ratio of the image $\widetilde{H S}_{k}$ and $R_{l}$. This allows integrating the contrast present in $R$ without creating missing objects in the multispectral image $\widetilde{H S}$. Due to this pixel by pixel multiplication, there can be an issue on the low values of $R_{l}$, which requires to manage the dynamic of the image, such that it does not exceed a given range.

\subsubsection{MRA by Laplacian Pyramid (MTF-GLP).}

This technique [Burt1983, Mallat1989] has some similarities with the previous one. However, this time a multiple low-pass filter $H_{\text {reso } i}$ at various resolutions (i.e., at various scales) is used. More precisely, the low-pass filters are typically Gaussian convolutions. Missing information at a given scale $i$ is injected into $\widehat{H S}_{\text {reso } i+1}$ thanks to information from $R$ at the resolution $i$. In our framework, the BSE image $R$ and the multispectral abundance maps image of the EDS image are both in a multi-resolution structure pyramid, as illustrated in Figure 5. Details are injected at each resolution, until the nominal resolution of the BSE image is reached. 


\subsection{An hybrid method: Guided PCA}

This technique [Liao2014, Kang2014] consists in first doing a PCA on the multispectral image $\widetilde{H S}$. Then, on the $d$ first principal components, a guided filter [He2013] is applied and nothing is done on the remaining $D-d$ bands. The rationale is based on the fact that the remaining bands correspond to "noise", and it would be useless to enhance the resolution of noise. Then a reversed PCA transform is made to obtain the original multispectral representation. The high resolution information from $R$ is included by the guided filter. For more details about this technique, see [Liao2014, Kang2014].

\section{Fusion of SEM information by Abundance Guided Bilateral Filtering (AGB)}

As we have discussed above, when a pansharpening technique is used in the image fusion context, the starting point is a low spatial resolution image $H S$, together with a high resolution "panchromatic" image $R$. Usually, the first step consists in upsampling $H S$, thanks to a basic bi-cubic interpolation to obtain the image $\widetilde{H S}$. Then, on this image at the nominal scale, the image enhancement is done, where the information is corrected by means of a particular pansharpening algorithm. The main issues with the previous techniques are that the CS approaches do not take into account the differences between the various components of the multimodal image, that is, the differences between the abundance maps themselves and the differences with the panchromatic image. In our case, there is a few correlation between the different EDX abundance maps. Moreover the MRA approaches do not consider the spectral link between the different abundance maps, since there is a finite quantity of material for each pixel (i.e., the sum of abundances at a given pixel is equal to 1).

In order to address these drawbacks, we propose an interpolation technique called abundance guided bilateral filter (AGB) by considering the relationships between the abundance maps. Thus, weights used in the interpolation would depend on both $R$ and $H S$. Our approach of interpolation uses bilateral filter [Tomasi1998] and more exactly its cross version [Eisemann2004]. By the way, the bilateral filter has already been used on other superresolution algorithms. Inspired by these works, we have chosen to improve the interpolation process which is a scaling process.

A bilateral filter is a nonlinear, edge-preserving denoising/regularizing operator for images. The intensity value at each pixel in an image is replaced by a weighted average of intensity values from nearby pixels. This weight can be based on a Gaussian distribution. Crucially, the weights depend not only on Euclidean distance between pixels on the grid, but also on their intensity (or more general radiometric) differences. Thanks to the fact that it uses spatial and range information, it preserves the edges, this is the reason why bilateral filter is used in super-resolution. 
Formally, the joint bilateral filter of an image $I$ guided by an image $F$ is defined as :

$$
I^{*}(x)=\frac{1}{W_{p}(x)} \sum_{x_{i} \in E} I\left(x_{i}\right) k\left(x, x_{i}\right),
$$

where the kernel weights are given by

$$
k\left(x, x_{i}\right)=f_{r}\left(\left\|F\left(x_{i}\right)-F(x)\right\|\right) g_{s}\left(\left\|x_{i}-x\right\|\right),
$$

and where the normalization term is just given as:

$$
W_{p}(x)=\sum_{x_{i} \in E} k\left(x, x_{i}\right),
$$

such that $f_{r}$ and $g_{s}$ represent respectively the range (or spectral) and spatial kernels of parameters $r$ and $s$. To simplify, we have chosen a Gaussian function for both kernels. However in our case we would like to consider the link between the different abundance maps. To handle this relationship, we need to define a guided function $\mathrm{F}$ as a global criterion. Hence, we used the level of mixing of pixels, which will be represented by an order map: an image of ordered levels of intensity. In a way the order map has a low value if the pixel contains a mixture of various different materials and a high value if it is almost pure, so it contains contribution of few materials.

Let us precise how this order map is computed using of the possible alternatives. The EDS spectrum at a position $i$ of the image is a vector $v_{i}$ which can be written as:

$$
v_{i}=\sum_{r=1}^{D} a_{r, i} m_{r}+n_{i},
$$

where $\left\{m_{r}\right\}_{r=1}^{D}$ represent the set of $D$ endmembers (spectral signature of the material), $a_{r, i}$ the abundance at vector $i$ of each endmember $r$, and $n_{i}$ an additive noise. This last term can be neglected. The nonnegative coefficients $a_{r}$ we consider are convex combination such that $\sum_{r=1}^{D} a_{r}=1$. By using this additional constraint, it is guaranteed to work on a $(D-1)$ simplex.

We have proposed in [Franchi2015] different techniques to calculate such order map on abundance map images. We adopt here an approach based on the notion of majorization [Marshall2010], which is a technique for ordering vectors of same sum. Let us consider two vectors $\mathbf{c}=\left(c_{1}, \ldots, c_{n}\right) \in \mathbb{R}^{n}$ and $\mathbf{d}=\left(d_{1}, \ldots, d_{n}\right) \in \mathbb{R}^{n}$, then we say that $C$ weakly majorizes $D$, written $\mathbf{c} \succ_{w} \mathbf{d}$, if and only if

$$
\left\{\begin{array}{l}
\sum_{i=1}^{k} c_{i}^{\downarrow} \geq \sum_{i=1}^{k} d_{i}^{\downarrow}, \forall k \in[1, n] \\
\sum_{i=1}^{n} c_{i}=\sum_{i=1}^{n} d_{i}
\end{array}\right.
$$

where $c_{i}^{\downarrow}$ and $d_{i}^{\downarrow}$ represent respectively the coordinates of $C$ and $D$ sorted in descending order. Majorization is not a partial order, since $\mathbf{c} \succ \mathbf{d}$ and $\mathbf{d} \succ \mathbf{c}$ do not imply $\mathbf{c}=\mathbf{d}$, it only implies that the components of each vector are equal, but not necessarily in the same order. 
Let us define a majorization-like partial order adapted to the abundances. A permutation $\tau_{i}$ of the coordinates of the vectors $v_{i}$ in the simplex is applied such that they are sorted in descending order. The majorization-like order $\leq_{\text {maj }}$ is defined as

$$
\begin{gathered}
v_{i} \leq \text { maj } v_{j} \Leftrightarrow \\
\left\{\begin{array}{l}
a_{\tau_{i}^{-1}(1), i}<a_{\tau_{j}^{-1}(1), j} \text { or } \\
a_{\tau_{i}^{-1}(1), i}=a_{\tau_{j}^{-1}(1), j} \text { and } a_{\tau_{i}^{-1}(2), i}<a_{\tau_{j}^{-1}(2), j} \text { or } \\
\vdots \\
a_{\tau_{i}^{-1}(1), i}=a_{\tau_{j}^{-1}(1), j} \text { and } \ldots \text { and } \\
a_{\tau_{i}^{-1}(R), i} \leq a_{\tau_{j}^{-1}(R), j}
\end{array}\right.
\end{gathered}
$$

This order map between pixels brings a global information: the materials entropy. Since a pixel that has a high value with this order is less mixed than the other one. Let us write $O$ the order map of the abundance images from the EDS image.

Figure 6 provides the corresponding $O$ image for dataset 1 at the resolution of $H S$, together the BSE image $R$ at the nominal resolution. Let us write $\widetilde{O}$ the order map image at the nominal resolution, where the value of all the missing pixels is put to zero.

We can now introduce the expression of the SEM fusion using the AGB filter:

$$
\widehat{H S}(x)=\frac{1}{W_{p}(x)} \sum_{x_{i} \in E} H S\left(x_{i}\right) \widehat{k}\left(x, x_{i}\right)
$$

where the AGB kernel is written as the product of two kernels

$$
\widehat{k}\left(x, x_{i}\right)=k_{\text {space }}\left(x, x_{i}\right) k_{E D X+B S E}\left(x, x_{i}\right)
$$

given by

$$
\begin{array}{r}
k_{\text {space }}\left(x, x_{i}\right)= \\
g_{s}\left(\left\|x_{i}-x\right\|\right) M\left(\widetilde{O}\left(x_{i}\right)\right), \\
k_{E D X+B S E}\left(x, x_{i}\right)= \\
g_{s}\left(\left|\widetilde{O}\left(x_{i}\right)-\widetilde{O}(x)\right|\right) g_{s}\left(\left|R\left(x_{i}\right)-R(x)\right|\right),
\end{array}
$$

with a typical normalization term:

$$
W_{p}(x)=\sum_{x_{i} \in E} \widehat{k}\left(x, x_{i}\right),
$$

$M$ is a mask that allows us to consider just the data on the values of abundance images available at the low resolution:

$$
M\left(\widetilde{O}\left(x_{i}\right)\right)= \begin{cases}1, & \text { if } \widetilde{O}\left(x_{i}\right) \neq 0 \\ 0, & \text { otherwise }\end{cases}
$$

and finally, $g_{s}$ is a Gaussian function of scale parameter $s$. 


\section{Results and discussion}

\subsection{Evaluation criteria of pansharpening algorithms}

The criteria used to evaluate the quality of the merged information are those conventionally considered in the context of the pansharpening literature. More precisely, we dealt with the following criteria:

C1: The spectral distortion between the enhanced multispectral image and the real multispectral image at the nominal resolution should be as small as possible. Or, in other terms, we would like to find the same materials for each pixel as the original image at high resolution;

C2: The spatial distortion between the enhanced multispectral image and the real one should not be too high.

Many alternative metrics can be used to quantify these two criteria [Loncan2015]. Let us formaly precise those that we have used. We write $\widehat{\widehat{H S}}$ the multispectral abundance EDS image at the same resolution that $R$, that has been provided by the sensor at high resolution. In a way it is the ground truth such that our enhanced images $\widehat{H S}$ should be compared to $\widehat{\widehat{H S}}$. Moreover, we denote by $\widehat{\widehat{\mathcal{M}}} \in M_{n, D}(\mathbb{R})$ and $\widehat{\mathcal{M}} \in M_{n, D}(\mathbb{R})$ the two matrices representing respectively $\widehat{\widehat{H S}}$ and $\widehat{H S}$, where $n$ is the total number of pixels (i.e., $n=N_{1} \times N_{2}$ ), and $D$ the number of abundance maps. We write by $\widehat{\mathcal{M}}_{i,:}, \forall i \in[1, n]$, a spectra of $\widehat{H S}$, and $\widehat{\mathcal{M}}:, k \forall \in[1, D]$ a map of $\widehat{H S}$. We have now all the notation to introduce the four parameters.

Cross correlation $(C C)$ is a measure that evaluate the spatial distortion defined as

$$
C C(\widehat{H S}, \widehat{\widehat{H S}})=\frac{1}{D} \sum_{k=1}^{D} \operatorname{CCS}\left(\widehat{\mathcal{M}}_{:, k}, \widehat{\widehat{\mathcal{M}}}_{:, k}\right),
$$

where

$$
\begin{aligned}
& \operatorname{CCS}\left(\widehat{\mathcal{M}}_{:, k}, \widehat{\widehat{\mathcal{M}}}_{:, k}\right)= \\
& \left(\sum_{i=1}^{n} \widehat{\mathcal{M}}_{i, k}-\mu_{\widehat{\mathcal{M}}_{i, k}}\right)\left(\sum_{i=1}^{n} \widehat{\widehat{\mathcal{M}}}_{i, k}-\mu_{\widehat{\mathcal{M}}_{i, k}}\right) \\
& \sqrt{\sum_{i=1}^{n}\left(\widehat{\mathcal{M}}_{i, k}-\mu_{\widehat{\mathcal{M}}_{i, k}}\right)^{2} \sum_{i=1}^{n}\left(\widehat{\widehat{\mathcal{M}}}_{i, k}-\mu_{\widehat{\mathcal{M}}_{i, k}}\right)^{2}},
\end{aligned}
$$

with $\mu_{\widehat{\mathcal{M}}_{i, k}}=n^{-1} \sum_{i=1}^{n} \widehat{\mathcal{M}}_{i, k}$ being the empirical mean. The $C C$ is optimal when it is close to 1 .

Spectral Angle Mapper (SAM) is a measure that assesses the spectral distortion by computing

$$
S A M(\widehat{H S}, \widehat{\widehat{H S}})=\frac{1}{n} \sum_{k=1}^{n} \widehat{S A M}\left(\widehat{\mathcal{M}}_{i,:}, \widehat{\widehat{\mathcal{M}}}_{i,:}\right)
$$


with

$$
\begin{array}{r}
\widehat{\operatorname{SAM}}\left(\widehat{\mathcal{M}}_{i,:}, \widehat{\widehat{\mathcal{M}}}_{i,:}\right)= \\
\arccos \left(\frac{<\widehat{\mathcal{M}}_{i,:}, \widehat{\widehat{\mathcal{M}}}_{i,:}>}{\left\|\widehat{\mathcal{M}}_{i,:}\right\|\left\|\widehat{\widehat{\mathcal{M}}}_{i,:}\right\|}\right),
\end{array}
$$

where $\langle\cdot, \cdot\rangle$ is the Euclidean inner product of vectors associated to the norm $L^{2}$, and where $\|\cdot\|$ is the norm $L^{2}$ of vectors. The $S A M$ is optimal when it is near to 0 .

Root mean squared error (RMSE) measures the mean residual error of fusion and is defined as:

$$
\operatorname{RMSE}(\widehat{H S}, \widehat{\widehat{H S}})=\frac{\|\widehat{\mathcal{M}}-\widehat{\widehat{\mathcal{M}}}\|_{F}}{n \cdot D}
$$

where $\|\cdot\|_{F}$ is the Frobenius norm of a matrix $A$, i.e., $\|A\|_{F}=\sqrt{\operatorname{trace}\left(A A^{t}\right)}$. The RMSE is optimal when it is near to zero.

Synthetic adimensional global error (ERGAS) offers an overall measure of the quality of an enhanced image. It is given by the expression:

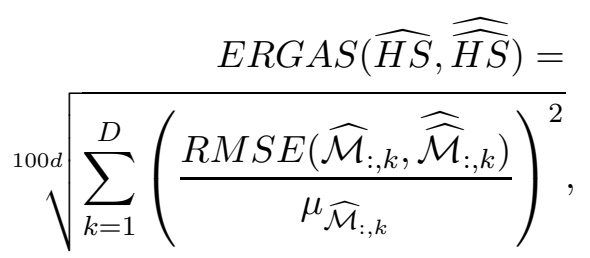

where $d$ is the ratio between the linear resolution of the BSE image $R$ and the abundances EDS image $H S$, i.e.,

$$
d=\frac{R \text {-linear spatial resolution }}{H S \text {-linear spatial resolution }}
$$

The ERGAS is optimal when close to 0.

We note that $\mathrm{C} 1$ and $\mathrm{C} 2$ are not enough to assess in our case the quality of the enhancement. Indeed the BSE images give not just access to the high resolution spatial information of the physical objects, but it provides other kind of information that can in theory allow us to have better result than just the EDX image at high resolution, such as the topographic shape of the sample. Thus we need to introduce a new criterion measuring the amount of information which is injected in the merged image:

C3: The injected information. Since the information provided by the various modalities can be relatively different, we would like to inject the useful one to improve segmentation and characterisation of the EDX image. 
In order to quantify this criterion, we proposed to use the cross correlation between the norm of gradient of the images. To calculate such norm of the image gradient, different techniques can be used. In our studies, we computed the morphological gradient [Soille2004]. More precisely, we have:

Cross correlation gradient $\left(C C_{g}\right)$ is a measure that evaluate the spatial distortion defined as

$$
\begin{array}{r}
\operatorname{CCg}_{g}(R, \widehat{\widehat{H S}})= \\
\frac{1}{D} \sum_{k=1}^{D} C C S\left(\mathcal{R} g, \widehat{\widehat{\mathcal{M}}} g_{:, k}\right),
\end{array}
$$

where $\mathcal{R} g$ represents the image gradient of the BSE image converted into a vector and $\widehat{\widehat{\mathcal{M}}} g_{:, k}$ is the image gradient of the enhanced abundance map at the nominal scale converted also into a vector.

An optimal enhancement method would be a compromise between the criterion $\mathrm{C} 3$ and the $\mathrm{C} 1$ and $\mathrm{C} 2$.

\subsection{Evaluation on dataset 1}

The EDS abundance maps of dataset 1 are provided in Figure 7. The results of the enhanced abundances $\widetilde{H S}$, obtained by the different techniques are provided in Figures 8, 9, 10, and 11 where the abundances are visualized as RGB color images. The results of our methods on dataset 1 are provided in Figure 12. Quantitative results for this case according to the five measures are presented in Table 1.

From these results, we note that CS techniques are good to inject $R$ on $\widehat{H S}$, while MRA techniques are good to increase the resolution. In addition, AGB and MTF-GLP present both a good compromise between these criteria.

\begin{tabular}{llllll} 
Techniques & CC & SAM & RMSE & ERGAS & $\mathbf{C C}_{\mathbf{g}}$ \\
\hline \hline GS & 0.783 & 22.6 & 27.7 & 16.8 & 0.69 \\
\hline PCA & 0.771 & 29.3 & 32.0 & 16.3 & 0.65 \\
\hline SFIM & 0.831 & 11.6 & 19.6 & 14.4 & 0.29 \\
\hline MTF GLP & $\mathbf{0 . 8 9 9}$ & 10.9 & 14.3 & $\mathbf{9 . 6}$ & $\mathbf{0 . 7 0}$ \\
\hline GFPCA & 0.840 & 12.2 & 15.2 & 12.2 & 0.55 \\
\hline AGB & $\mathbf{0 . 9 0}$ & $\mathbf{9 . 5}$ & $\mathbf{1 1 . 2}$ & 9.35 & 0.59 \\
\hline
\end{tabular}

Table 1: Comparison of pansharpening algorithms for enhanced EDS abundance images of dataset 1 .

\subsection{Evaluation on dataset 2}

In the case of this multimodal SEM dataset, which has a higher level of noise, we try to inject the BSE image $R$, depicted in Figure 13, to increase the resolution of the EDS abundance 
maps provided in Figure 14, and also to denoise the images. The results for our ABG method are given in Figure 15.

For comparaison, Figures 16 and 17 provide a visualization of the results obtained using the other methods. Quantitative results are given in Table 2, which lead to similar conclusions as for dataset 1 .

\begin{tabular}{llllll} 
Techniques & CC & SAM & RMSE & ERGAS & CC $_{\mathbf{g}}$ \\
\hline \hline GS & 0.23 & 16.3 & 1.6 & 20.3 & 0.66 \\
\hline PCA & 0.17 & 17.4 & 1.6 & 20.6 & $\mathbf{0 . 6 7}$ \\
\hline SFIM & 0.14 & 15.9 & 1.74 & 27.9 & 0.17 \\
\hline MTF GLP & $\mathbf{0 . 3 0}$ & 15.8 & $\mathbf{1 . 4}$ & $\mathbf{2 0 . 1}$ & 0.61 \\
\hline GFPCA & 0.26 & 15.9 & 1.5 & 20.4 & 0.31 \\
\hline AGB & $\mathbf{0 . 3 0}$ & $\mathbf{1 5 . 7}$ & $\mathbf{1 . 4}$ & $\mathbf{2 0 . 1}$ & 0.45 \\
\hline
\end{tabular}

Table 2: Comparison of pansharpening algorithms for enhanced EDS abundance images of dataset 2 .

\subsection{Evaluation on simulated dataset}

By means of the Monte Carlo software based on PENELOPE, we calculated various simulated datasets with different levels of noise and different resolutions. So first we have a dataset represented in Figure 2. It represents the sample at the full resolution without noise, thus it corresponds to the ground truth. The size of this image is $1024 \times 1024 \times 2048$. Then we simulate other EDX images of the same sample at resolution 4 times smaller than the original. The size of these images is therefore $256 \times 256 \times 2048$. First we extract the 7 abundance maps of these images and perform the fusion techniques. The results of the different algorithms are gathered in Table 3. Since we know the ground truth, for each pixel the most important materials that are present are known (see Figure 18). Hence after having performed the fusion techniques, for each pixel we extract the most significant material. This imply that, each pixel is represented by a number between 1 and 7 . Then these images can be seen as the results of a classification. To evaluate the performance we used the overall accuracy $(\mathrm{OA})$ which is defined as the percentage of pixels well classified on the whole image, and the average accuracy (AA) which represents mean of the accuracy of each class. These grey scale images are represented in Figure 18. One can see the results with the different levels of noise in Table 4. Our proposed ABG method and GFPCA give the best accuracies, even with high noise levels. We have represented the result of our method on 19. Thanks the figures and the tables one can see that our method is able to increase the resolution.

\section{Conclusion}

In this article, we have conducted a qualitative and quantitative evaluation of different algorithms of fusion of information applied on SEM images. Thanks to these algorithms we merged EDX and backscattered electrons SEM images. We compared global and local state 


\begin{tabular}{llllll} 
Techniques & CC & SAM & RMSE & ERGAS & CCg \\
\hline \hline GS & 0.065 & 26.72 & 0.51 & 445.61 & 0.12 \\
\hline PCA & 0.046 & 26.52 & 0.62 & 461.6 & 0.20 \\
\hline SFIM & 0.15 & 24.66 & 0.61 & 365.3 & 0.045 \\
\hline MTF GLP & 0.19 & 24.0502 & 0.42 & 266.4 & 0.0763 \\
\hline GFPCA & 0.29 & $\mathbf{1 6 . 3 9}$ & $\mathbf{0 . 2 1 8}$ & 439.4 & 0.1766 \\
\hline AGB & $\mathbf{0 . 3 6}$ & $\mathbf{1 6 . 3 0}$ & $\mathbf{0 . 2 1 7}$ & $\mathbf{4 2 0 . 6}$ & $\mathbf{0 . 3 5 2 9}$ \\
\hline
\end{tabular}

Table 3: Comparison of pansharpening algorithms for enhanced EDS abundance images on simulated SEM image, forcing level 1.

\begin{tabular}{llll} 
Techniques & forcing level & OA & AA \\
\hline \hline GS & 1 & 71.0 & 22.8 \\
\hline PCA & 1 & 71.0 & 20.8 \\
\hline SFIM & 1 & 70.0 & 33.3 \\
\hline MTF GLP & 1 & 70.8 & 33.4 \\
\hline GFPCA & 1 & $\mathbf{7 8 . 2}$ & $\mathbf{3 6 . 9}$ \\
\hline AGB & 1 & $\mathbf{7 8 . 9}$ & $\mathbf{3 6 . 9}$ \\
\hline GS & 10 & 74.9 & 23.0 \\
\hline PCA & 10 & 75.3 & 21.5 \\
\hline SFIM & 10 & 77.8 & 34.4 \\
\hline MTF GLP & 10 & 77.9 & 34.4 \\
\hline GFPCA & 10 & $\mathbf{7 9 . 2}$ & $\mathbf{3 6 . 5}$ \\
\hline AGB & 10 & $\mathbf{7 9 . 1}$ & $\mathbf{3 6 . 7}$ \\
\hline GS & 100 & 75.1 & 22.4 \\
\hline PCA & 100 & 75.8 & 22.6 \\
\hline SFIM & 100 & 78.3 & 35.3 \\
\hline MTF GLP & 100 & $\mathbf{7 9 . 1}$ & 35.5 \\
\hline GFPCA & 100 & $\mathbf{7 9 . 2}$ & $\mathbf{3 6 . 5}$ \\
\hline AGB & 100 & $\mathbf{7 9 . 2}$ & $\mathbf{3 6 . 8}$ \\
\hline
\end{tabular}

Table 4: Comparison of pansharpening algorithms for enhanced EDS abundance images on simulated SEM image, for different forcing levels.

of the art techniques and proposed an innovative one based on a guided bilateral filter. We also wrote a program based on the PENELOPE package to simulate large SEM-EDX spectral maps.

Thanks to these simulated and real SEM images, we evaluated the performances of the different techniques according to criteria defined for pansharpening problem. We also proposed one new criterion that is more adapted for the SEM segmentation problem. The accuracy of the pansharpening methods was assessed, and our proposed method was always found to be one of the best, together with the GFPCA. Our proposed algorithm performs well even on very noisy EDX images. Thanks to this fusion of information, we can increase the speed of the imaging process, since we need fewer pixels. A way to improve the compression of this technique might be to consider pixels at given location, where the location is guided thanks to the backscattered image. By doing that, we would first take the backscattered image then have a look at the keypoints localizations, and then take the EDX image just at those points. Another way to improve this technique might be to consider the secondary electrons image. In addition, thanks to the developed formalism, it can easily be taken into account. In a broader perspective, the proposed guided bilateral filter could be used for other multispectral image pansharpening than SEM based ones. 


\section{Acknownledgement}

Florent Moreau is gratefully acknowledged for the acquisition of experimental SEM-EDX maps. 


\section{List of figures}

- Figure 1 : Multimodal SEM image acquisition. In the red square ( a,b,c,d) we represented 4 abundances. In the blue square (e) we represented the backscattered image and in the green square (f) the secondary electron image. As one can notice each modality brings different information, that are perfectly registred in our experimental set up. Our goal is to merge the SEM backscattered (green square) and SEM EDX modality (red square).

- Figure 2: Ground truth of the simulated multimodal SEM image.

- Figure 3 : Simulated backscattered electron image.

- Figure 4 : Simulated Al energy channel in (a), Co energy channel in (b), Fe energy channel in (c), Ni energy channel in (d), Si energy channel in (e), Pt energy channel ( the first peak) in (f).

- Figure 5: Model of a hierarchical decomposition of the information. The algorithm takes as input image $\widehat{H S}_{\text {reso } 0}$ and increases its resolution to build $\widehat{H S}_{\text {reso } 1}$, and so one up to $\widehat{H S}$.

- Figure 6 : The two images used for the fusion of SEM information by AGB filter.

- Figure 7 : The six EDS abundance maps of SEM dataset 1 at the nominal resolution.

- Figure 8: RGB color image from abundances 1,2,4 of the dataset 1 for the different pansharpening techniques. The input image has been simplified by applying a dilation for visualization purpose.

- Figure 9: Zoom of the RGB color image from abundances 1,2,4 of the dataset 1 for the different pansharpening techniques. The input image has been simplified by applying a dilation for visualization purpose.

- Figure 10 : RGB color image from abundances 3,5,6 of the dataset 1 for the different pansharpening techniques. The input image has been simplified by applying a dilation for visualization purpose.

- Figure 11 : Zoom of the RGB color image from abundances 3,5,6 of the dataset 1 for the different pansharpening techniques. The input image has been simplified by applying a dilation for visualization purpose.

- Figure 12 : Six enhanced EDS abundance maps by means of ABG method of SEM dataset 1 at the nominal resolution.

- Figure 13 : BSE image of SEM dataset 2. 
- Figure 14 : Four EDS abundance maps of SEM dataset 2 at the nominal resolution.

- Figure 15 : Four enhanced EDS abundance maps by means of ABG method of SEM dataset 2 at the nominal resolution.

- Figure 16 : RGB color image from abundances 2,3,4 of the dataset 2 for the different pansharpening techniques. The input image has been simplified by applying a dilation for visualization purpose.

- Figure 17 : Zoom of the RGB color image from abundances 2,3,4 of the dataset 2 for the different pansharpening techniques. The input image has been simplified by applying a dilation for visualization purpose.

- Figure 18 : Extraction of the most abundant material at each pixel for the different pansharpening techniques.

- Figure 19 : Six enhanced EDS abundance maps by means of ABG method of SEM simulated dataset at the nominal resolution. 


\section{References}

[Wald1999] L. Wald Some terms of reference in data fusion, IEEE Trans. Geosci. Remote Sens., vol. 37, no. 3, pp.1190 -1193 1999

[Piella2003] G. Piella A general framework for multiresolution image fusion: From pixels to regions, Inf. Fusion, vol. 4, no. 4, pp.259 -280 2003

[Loncan2015] L. Loncan, L. B. Almeida, J. M. Bioucas-Dias, X. Briottet, J. Chanussot, N. Dobigeon, \& N. Yokoya, (2015). Hyperspectral pansharpening: a review. arXiv preprint arXiv:1504.04531.

[Vivone2015] G. Vivone, L. Alparone, J. Chanussot, M. Dalla Mura, A. Garzelli, G. A. Licciardi, \& L. Wald, (2015). A critical comparison among pansharpening algorithms. Geoscience and Remote Sensing, IEEE Transactions on, 53(5), 2565-2586.

[Pearson1901] K. Pearson, (1901). LIII. On lines and planes of closest fit to systems of points in space. The London, Edinburgh, and Dublin Philosophical Magazine and Journal of Science, 2(11), 559-572.

[Hotelling1993] H. Hotelling, (1933). Analysis of a complex of statistical variables into principal components. Journal of educational psychology, 24(6), 417.

[Chavez1991] P. Chavez, S. C. Sides, \& J. A. Anderson, (1991). Comparison of three different methods to merge multiresolution and multispectral data- Landsat TM and SPOT panchromatic. Photogrammetric Engineering and remote sensing, 57(3), 295-303.

[Thomas2008] C. Thomas, T. Ranchin, L. Wald, \& J. Chanussot, (2008). Synthesis of multispectral images to high spatial resolution: A critical review of fusion methods based on remote sensing physics. Geoscience and Remote Sensing, IEEE Transactions on, 46(5), 1301-1312.

[King2001] R. L. King, \& J. Wang, (2001). A wavelet based algorithm for pan sharpening Landsat 7 imagery. In Geoscience and Remote Sensing Symposium, 2001. IGARSS'01. IEEE 2001 International (Vol. 2, pp. 849-851). IEEE.

[Wortmann2009] T. Wortmann, Fusion of AFM and SEM Scans. In : Optomechatronic Technologies, 2009. ISOT 2009. International Symposium on. IEEE, 2009. p. 40-45.

[Milillo2008] T. M. Milillo, \& J. AGardella Jr. (2008). Spatial Analysis of Time of FlightSecondary Ion Mass Spectrometric Images by Ordinary Kriging and Inverse Distance Weighted Interpolation Techniques. Analytical chemistry, 80(13), 4896-4905.

[Angelov20016] T. Angelov, A. Ahmad, E. Guliyev, A. Reum, I. Atanasov, T. Ivanov, \& C. Lenk, (2016). Six-axis AFM in SEM with self-sensing and self-transduced cantilever 
for high speed analysis and nanolithography. Journal of Vacuum Science \& Technology B, Nanotechnology and Microelectronics: Materials, Processing, Measurement, and Phenomena, 34(6).

[Reimer1998] L. Reimer, (1998). Scanning electron microscopy: physics of image formation and microanalysis. Springer Verlag, Heidelberg, second edition.

[Sempau1997] J. Sempau, E. Acosta, J. Baro, J. M. Fernandez-Varea, \& F. Salvat,(1997). An algorithm for Monte Carlo simulation of coupled electron-photon transport. Nuclear Instruments and Methods in Physics Research Section B: Beam Interactions with Materials and Atoms, 132(3), 377-390.

[Sempau2003] J. Sempau, J. M. Fernandez-Varea, E. Acosta, \& F. Salvat,(2003). Experimental benchmarks of the Monte Carlo code PENELOPE. Nuclear Instruments and Methods in Physics Research Section B: Beam Interactions with Materials and Atoms, 207(2), 107-123.

[Salvat2006] F. Salvat, J.M. Fernandez-Varea \& J. Sempau,(2006, July). PENELOPE-2006: A code system for Monte Carlo simulation of electron and photon transport NEA Data Bank NEA-15 25 PENELOPE2011, http://www.oecd-nea.org/tools/abstract/detail/nea1525. In Workshop Proceedings (Vol. 4, p. 7).

[Limandri2013] S. Limandri, G. Bernardi,\& S. Suarez, (2013). Experimental study of the efficiency of a SDD X-ray detector by means of PIXE spectra. X-Ray Spectrometry, $42(6), 487-492$.

[Laben2000] C. A. Laben, \& B. V. Brower,(2000).Process for enhancing the spatial resolution of multispectral imagery using pan-sharpening, U.S. Patent No. 6,011,875. Washington, DC: U.S. Patent and Trademark Office.

[Ranchin2000] T. Ranchin, \& L. Wald, (2000). Fusion of high spatial and spectral resolution images: the ARSIS concept and its implementation. Photogrammetric Engineering and Remote Sensing, 66(1), 49-61.

[Scholze2009] F. Scholze, \& M. Procop, (2009). Modelling the response function of energy dispersive X-ray spectrometers with silicon detectors. X-Ray Spectrometry, 38(4), 312321.

[Bauer1978] R. Bauer, \& R. Rick, (1978). Computer analysis of X-ray spectra (EDS) from thin biological specimens. X-Ray Spectrometry, 7(2), 63-69.

[Tu2001] T. M. Tu, S. C. Su, H. C. Shyu,\& P. S. Huang, (2001). A new look at IHS-like image fusion methods. Information fusion, 2(3), 177-186. 
[Liu2000] J. G. Liu, (2000). Smoothing filter-based intensity modulation: a spectral preserve image fusion technique for improving spatial details. International Journal of Remote Sensing, 21(18), 3461-3472.

[Mallat1989] S. Mallat, (1989). A theory for multiresolution signal decomposition: the wavelet representation. Pattern Analysis and Machine Intelligence, IEEE Transactions on, 11(7), 674-693.

[Burt1983] P. J. Burt, \& E. H. Adelson,(1983). The Laplacian pyramid as a compact image code. Communications, IEEE Transactions on, 31(4), 532-540.

[He2013] K. He, J. Sun, \& X. Tang,(2013). Guided image filtering. Pattern Analysis and Machine Intelligence, IEEE Transactions on, 35(6), 1397-1409.

[Liao2014] W. Liao, X. Huang, F. Van Coillie, S. Gautama, A. Pizurica, W. Philips, \& D. Tuia, Processing of multiresolution thermal hyperspectral and digital color data: Outcome of the 2014 IEEE GRSS data fusion contest.

[Kang2014] X. Kang, S. Li, \& J. A. Benediktsson, (2014). Spectral-spatial hyperspectral image classification with edge-preserving filtering. Geoscience and Remote Sensing, IEEE Transactions on, 52(5), 2666-2677.

[Marshall2010] A.W. Marshall, I. Olkin, \& B.C. Arnold, (2010). Inequalities: Theory of Majorization and Its Applications: Theory of Majorization and Its Applications, Springer.

[Tomasi1998] C. Tomasi, \& R. Manduchi, (1998, January). Bilateral filtering for gray and color images. In Computer Vision, 1998. Sixth International Conference on (pp. 839-846). IEEE.

[Eisemann2004] E. Eisemann, \& F. Durand, (2004). Flash photography enhancement via intrinsic relighting. ACM transactions on graphics (TOG), 23(3), 673-678.

[Franchi2015] G. Franchi, \& J. Angulo, (2015). Ordering on the probability simplex of endmembers for hyperspectral morphological image processing. In Mathematical Morphology and Its Applications to Signal and Image Processing (pp. 410-421). Springer International Publishing.

[Soille2004] P. Soille, (2004). Morphological Image Analysis - Principles and Applications, Springer. 


\section{List of Figures}

1 Multimodal SEM image acquisition. In the red square (a,b,c,d) we represented 4 abundances. In the blue square (e) we represented the backscattered image and in the green square $(\mathrm{f})$ the secondary electron image. . . . . . . . . . . . 23

2 Ground truth of the simulated multimodal SEM image. . . . . . . . . . . . . 24

3 Simulated backscattered electron image. . . . . . . . . . . . . . . 25

4 (a) The pixelwise mean image of the simulated EDX image $(256 \times 256, F=1)$.

(b) Simulated $\mathrm{Al} \mathrm{K \alpha}$ energy channel. . . . . . . . . . . . . . . . . . . . 26

5 Model of a hierarchical decomposition of the information. . . . . . . . . 27

6 The two images used for the fusion of SEM information by AGB filter. . . . . 28

7 The six EDS abundance maps of SEM dataset 1 at the nominal resolution. . $\quad 29$

8 RGB color image from abundances 1,2,4 of the dataset 1 for the different pansharpening techniques. . . . . . . . . . . . . . 30

9 Zoom of the RGB color image from abundances 1,2,4 of the dataset 1 for the different pansharpening techniques. . . . . . . . . . . . . . . . 31

10 RGB color image from abundances $3,5,6$ of the dataset 1 for the different pansharpening techniques. . . . . . . . . . . . . .

11 Zoom of the RGB color image from abundances 3,5,6 of the dataset 1 for the different pansharpening techniques. . . . . . . . . . . . . . .

12 Six enhanced EDS abundance maps by means of ABG method of SEM dataset 1 at the nominal resolution. . . . . . . . . . . . . . . . . . . . 34

13 BSE image of SEM dataset 2. . . . . . . . . . . . . . . . . 35

14 Four EDS abundance maps of SEM dataset 2 at the nominal resolution. . . . 36

15 Four enhanced EDS abundance maps by means of ABG method of SEM dataset 2 at the nominal resolution. . . . . . . . . . . . . . . 37

16 RGB color image from abundances $2,3,4$ of the dataset 2 for the different pansharpening techniques. . . . . . . . . . . . . . . 38

17 Zoom of the RGB color image from abundances 2,3,4 of the dataset 2 for the different pansharpening techniques. . . . . . . . . . . . . .

18 Extraction of the most abundant material at each pixel for the different pansharpening techniques. . . . . . . . . . . . . . . .

19 Six enhanced EDS abundance maps by means of ABG method of SEM simulated dataset at the nominal resolution. . . . . . . . . . . . .

4
6 
Figure 1: Multimodal SEM image acquisition. In the red square ( a,b,c,d) we represented 4 abundances. In the blue square (e) we represented the backscattered image and in the green square (f) the secondary electron image. 
Figure 2: Ground truth of the simulated multimodal SEM image. 
Figure 3: Simulated backscattered electron image. 
(a) (b)

Figure 4: (a) The pixelwise mean image of the simulated EDX image $(256 \times 256, F=1)$. (b) Simulated Al K $\alpha$ energy channel. 
Figure 5: Model of a hierarchical decomposition of the information. 
Figure 6: The two images used for the fusion of SEM information by AGB filter. 
abundance 1 abundance 2

abundance 3 abundance 4

abundance 5 abundance 6

Figure 7: The six EDS abundance maps of SEM dataset 1 at the nominal resolution. 


$\begin{array}{cc}\text { Reference } & \text { Input image } \\ \text { GS method } & \text { PCA method } \\ \text { SFIM method } & \text { MTF GLP method } \\ \text { GFPCA method } & \text { AGB method }\end{array}$

Figure 8: RGB color image from abundances 1,2,4 of the dataset 1 for the different pansharpening techniques. 


$\begin{array}{cc}\text { Reference } & \text { Input image } \\ \text { GS method } & \text { PCA method } \\ \text { SFIM method } & \text { MTF GLP method } \\ \text { GFPCA method } & \text { AGB method }\end{array}$

Figure 9: Zoom of the RGB color image from abundances 1,2,4 of the dataset 1 for the different pansharpening techniques. 


$\begin{array}{cc}\text { Reference } & \text { Input image } \\ \text { GS method } & \text { PCA method } \\ \text { SFIM method } & \text { MTF GLP method } \\ \text { GFPCA method } & \text { AGB method }\end{array}$

Figure 10: RGB color image from abundances 3,5,6 of the dataset 1 for the different pansharpening techniques. 


$\begin{array}{cc}\text { Reference } & \text { Input image } \\ \text { GS method } & \text { PCA method } \\ \text { SFIM method } & \text { MTF GLP method } \\ \text { GFPCA method } & \text { AGB method }\end{array}$

Figure 11: Zoom of the RGB color image from abundances 3,5,6 of the dataset 1 for the different pansharpening techniques. 


\section{Al abundance Co abundance}

Figure 12: Six enhanced EDS abundance maps by means of ABG method of SEM dataset 1 at the nominal resolution. 
Figure 13: BSE image of SEM dataset 2. 
abundance 1 abundance 2

abundance 3 abundance 4

Figure 14: Four EDS abundance maps of SEM dataset 2 at the nominal resolution. 
abundance 1 abundance 2

abundance 3 abundance 4

Figure 15: Four enhanced EDS abundance maps by means of ABG method of SEM dataset 2 at the nominal resolution. 


$\begin{array}{cc}\text { Reference } & \text { Input image } \\ \text { GS method } & \text { PCA method } \\ \text { SFIM method } & \text { MTF GLP method } \\ \text { GFPCA method } & \text { AGB method }\end{array}$

Figure 16: RGB color image from abundances $2,3,4$ of the dataset 2 for the different pansharpening techniques. 


$\begin{array}{cc}\text { Reference } & \text { Input image } \\ \text { GS method } & \text { PCA method } \\ \text { SFIM method } & \text { MTF GLP method } \\ \text { GFPCA method } & \text { AGB method }\end{array}$

Figure 17: Zoom of the RGB color image from abundances 2,3,4 of the dataset 2 for the different pansharpening techniques. 


\section{Reference GS method \\ PCA method SFIM method \\ MTF GLP method GFPCA method \\ AGB method}

Figure 18: Extraction of the most abundant material at each pixel for the different pansharpening techniques. 
Figure 19: Six enhanced EDS abundance maps by means of ABG method of SEM simulated dataset at the nominal resolution. 